\title{
The influence of vector-borne disease on human history: socio-ecological mechanisms
}

Tejas Athni ${ }^{1}$, Marta Shocket ${ }^{1}$, Lisa Couper ${ }^{1}$, Nicole Nova ${ }^{1}$, Iain Caldwell ${ }^{2}$, Jamie Caldwell $^{1}$, Jasmine Childress ${ }^{3}$, Marissa Childs ${ }^{1}$, Giulio De Leo ${ }^{4}$, Devin Kirk ${ }^{1}$, Andrew MacDonald $^{3}$, Kathryn Olivarius ${ }^{1}$, David Pickel ${ }^{1}$, Steven Roberts ${ }^{1}$, Olivia Winokur ${ }^{5}$, Hillary Young $^{3}$, Julian Cheng ${ }^{1}$, Elizabeth Grant ${ }^{1}$, Patrick Kurzner ${ }^{1}$, Saw Kyaw ${ }^{1}$, Bradford Lin ${ }^{1}$, Ricardo Lopez ${ }^{1}$, Diba Massihpour ${ }^{1}$, Erica Olsen ${ }^{1}$, Maggie Roache ${ }^{1}$, Angie Ruiz ${ }^{1}$, Emily Schultz ${ }^{1}$, Muskan Shafat ${ }^{1}$, Rebecca Spencer ${ }^{1}$, Nita Bharti ${ }^{6}$, and Erin Mordecai ${ }^{1}$

${ }^{1}$ Stanford University

${ }^{2}$ James Cook University

${ }^{3}$ University of California Santa Barbara

${ }^{4}$ Stanford Universoty

${ }^{5}$ University of California Davis

${ }^{6}$ Pennsylvania State University

November 12, 2020

\begin{abstract}
Vector-borne diseases (VBDs) are embedded within complex socio-ecological systems. While research has traditionally focused on direct effects of VBDs on morbidity and mortality, it is increasingly clear that VBD impacts are much more pervasive, dynamically linked to feedbacks between environmental conditions, vector ecology, disease burden, and societal responses that drive transmission. VBDs have had profound influence on human history via mechanisms that include: (1) killing or debilitating large numbers of people, with direct demographic and population-level impacts; (2) differentially affecting populations based on prior history of disease exposure, immunity, and resistance; (3) being weaponized to promote or justify existing hierarchies of power, colonialism, racism, classism, and sexism; (4) catalyzing changes in ideas, institutions, infrastructure, technologies, and social practices in efforts to control disease outbreaks; and (5) changing human relationships with the land and environment. We use historical and archaeological evidence interpreted through an ecological lens to illustrate how four major VBDs have shaped society and culture: plague, malaria, yellow fever, and trypanosomiasis. By comparing across diseases, time periods, and geographies, this review highlights the enormous scope and variety of mechanisms by which VBDs have influenced human history from the age of early Homo sapiens to the modern context.
\end{abstract}

\section{Hosted file}

The influence of vector-borne disease on human history- socio-ecological mechanisms (1).pdf available at https://authorea.com/users/330215/articles/492530-the-influence-of-vectorborne-disease-on-human-history-socio-ecological-mechanisms 

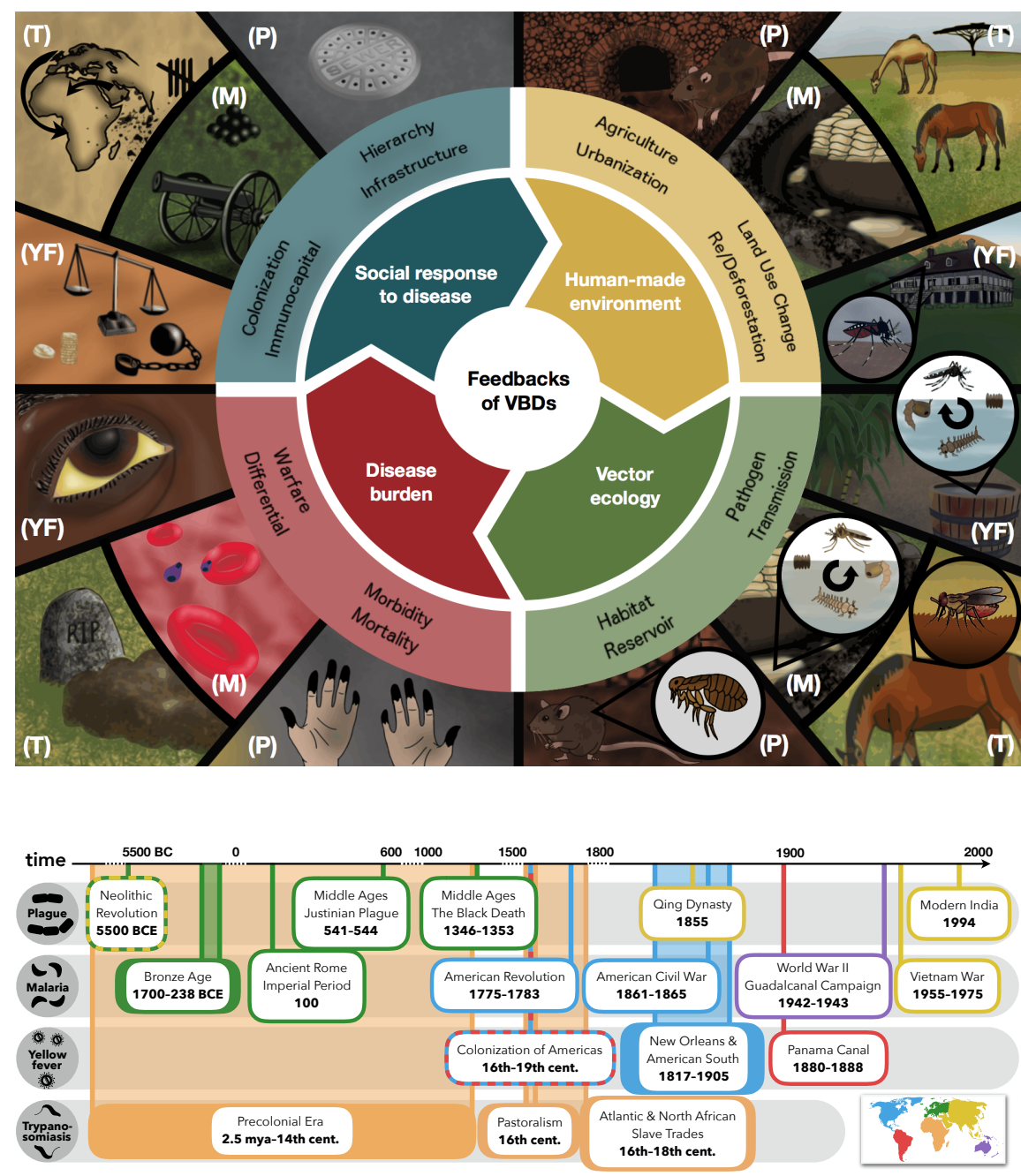

\section{Hosted file}

Table1.pdf available at https://authorea.com/users/330215/articles/492530-the-influence-ofvector-borne-disease-on-human-history-socio-ecological-mechanisms

\section{Hosted file}

Table2.pdf available at https://authorea.com/users/330215/articles/492530-the-influence-ofvector-borne-disease-on-human-history-socio-ecological-mechanisms 